\title{
Humeanismo y leyes de la naturaleza: alcance y límites
}

\author{
Humeanism and laws of nature: scope and limits \\ Cristian Soto \\ Departamento de Filosofía, Universidad de Chile, Chile \\ cssotto@uchile.cl
}

\begin{abstract}
Resumen
El humeanismo nomológico ha llegado a constituir un programa de investigación que acoge numerosas variaciones sobre un mismo tema, a saber, la idea de que las leyes son enunciados acerca de regularidades que observamos en la naturaleza. Luego de revisar la formulación inicial del humeanismo nomológico en la crítica de Hume a la idea de conexión necesaria, el presente trabajo examina la reformulación lewisiana basada en la superveniencia humeana y la teoría del mejor sistema. Examinaremos, a este respecto, tres límites que la literatura ha identificado en la propuesta humeana: su falta de sustento en las teorías físicas, su circularidad explicativa y su antropomorfismo, tres problemas que han intentado ser superados por versiones recientes del humeanismo (Jaag y Loew 2020, Loewer 2004 y Massimi 2018). Finalmente, sostendremos que el humeanismo nomológico falla al no dar cuenta de una noción adecuada de modalidad para las leyes de la naturaleza. Este último sigue siendo un desafío actual para el empirismo en filosofía de leyes físicas.
\end{abstract}

Palabras clave: modalidad, superveniencia, mejor sistema, necesidad, contingencia.

\begin{abstract}
Nomological Humeanism has developed into a research program encompassing several variations on a single theme, namely, the view that laws are statements about regularities that we find in nature. After briefly revisiting an early form of nomological Humeanism in Hume's critique of the idea of necessary connection, this article critically examines Lewis' two-fold approach based on Humean supervenience
\end{abstract}

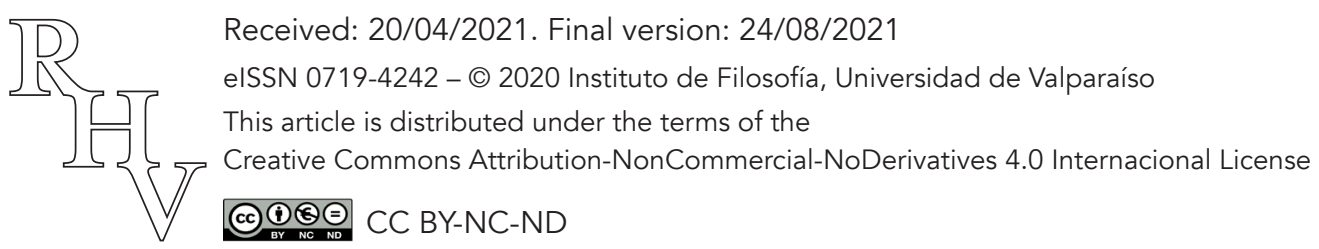


and the best system account. We shall point out three limits of nomological Humeanism, which are widely recognized in the literature: its inadequacy in view of physical theories, its explanatory circularity, and its purported anthropomorphism, all of which advocates of nomological Humeanism have attempted to overcome $\mathrm{Hu}-$ meanism (Jaag y Loew 2020, Loewer 2004 y Massimi 2018). Lastly, we will argue that nomological Humeanism fails to provide a suitable notion of modality for laws of nature. This latter issue continues to represent a live challenge for empiricism in the philosophy of physical laws.

Keywords: modality, supervenience, best system, necessity, contingency.

\section{Introducción}

Una de las concepciones dominantes en filosofía de las leyes de la naturaleza tiene su origen en el empirismo de David Hume y su crítica acérrima a las ontologías de poderes causales y conexiones necesarias que abundaban en el aristotelismo escolástico y en el mecanicismo temprano de Descartes y Newton, respectivamente. Aunque Hume no propuso una teoría de leyes de la naturaleza, su trabajo ofrece los cimientos para una ontología que clama por una concepción alternativa acerca de las leyes, distanciándose de las ideas de la filosofía natural inmediatamente precedente. Hume sostiene que el mundo está compuesto de particulares cuyas naturalezas son amodales, vale decir, que no involucran posibilidad o necesidad alguna más allá de la mera actualidad de sus instanciaciones. Todo lo que encontramos es que hay unos aquí y otros allá, algunos instanciando cierta conjunción espacial o sucesión temporal más o menos constante, pero en ningún caso accedemos a poderes causales o conexiones necesarias que, según la filosofía natural precedente, fundamentan las regularidades que observamos. Pues bien, si nuestro conocimiento se limita a la observación de regularidades espaciotemporales que instancian los particulares amodales del universo humeano, ¿qué lugar queda para las leyes de la naturaleza?

En el presente capítulo, revisaremos algunas ideas centrales de la filosofía de Hume, examinando lo que llamaremos el desafío humeano acerca de las leyes de la naturaleza (sección 2). Tras revisar elementos de la epistemología humeana y su concepción de la causalidad, mostraremos que los manuscritos de Hume permiten esbozar la llamada teoría de la regularidad de las leyes de la naturaleza. A partir de ello, mostraremos que el desafío humeano delinea algunos de los problemas claves que posteriormente serán desarrollados por el humeanismo contemporáneo, a saber, el problema de la ontología de las leyes de la naturaleza, su modalidad y la dirección de la explicación.

La sección 3 examinará las tesis de la superveniencia humeana y la teoría del mejor sistema, ambas desarrolladas por David Lewis, quien concibe su proyecto filosófico como una defensa y elaboración del proyecto de Hume. La tesis de la superveniencia humeana 
elabora un mosaico ontológico compuesto de patrones de instanciación de propiedades intrínsecas perfectamente naturales y sus relaciones espaciotemporales. Se concibe que tal mosaico es amodal, y que sobre él supervienen las regularidades que observamos y que aparecen en las formulaciones de nuestras teorías. Lewis da un paso más allá de la teoría de Hume al introducir la teoría del mejor sistema: si bien la teoría de la regularidad se limita a reconocer que observamos regularidades en la naturaleza (y por ende una teoría de la regularidad de las leyes de la naturaleza se limita a sostener que las leyes son enunciados de meras regularidades), la teoría del mejor sistema añadirá los criterios de simplicidad y robustez informativa, junto al mejor balance entre ambos criterios, que nos permitirían identificar aquellas enunciados que ocupan el lugar de axiomas en un sistema deductivo, i.e., las leyes de la naturaleza. De esta manera, la teoría del mejor sistema acerca de las leyes de la naturaleza se presenta como un refinamiento de la teoría de la regularidad nomológica.

El desarrollo del humeanismo acerca de las leyes de la naturaleza, aunque promisorio, no está libre de problemas (sección 4). Argumentaremos que no es claro en qué medida las tesis humeanas, en particular las de Lewis, encuentran fundamento en nuestras mejores teorías científicas; igualmente, resulta problemático determinar cómo las leyes de la naturaleza, dentro del marco teórico del mejor sistema, podrían explicar los patrones que las instancian, si de antemano se ha sostenido que son estas mismas instancias las que dan lugar a (o explican) las leyes de la naturaleza que tenemos en el universo humeano; asimismo, mostraremos que, aunque los criterios de simplicidad y robustez informativa representan un refinamiento de la teoría de la regularidad, estos criterios arriesgan introducir apreciaciones antropomórficas en nuestra concepción de las leyes de la naturaleza. Aunque no se trata de problemas que echen por tierra el humeanismo nomológico, ellos han motivado desarrollos ulteriores de las propuestas humeanas, ofreciendo respuestas posibles a estos problemas.

La sección 5 desarrollará uno de los problemas centrales para cualquier propuesta acerca de leyes de la naturaleza, a saber, su estatus modal. Este es uno de los consensos más ampliamente aceptados en la literatura: si hay leyes de la naturaleza, ellas tienen que gozar de un estatus modal, permitiéndonos explicar no solamente las cosas que han sido o son el caso, sino también cosas que podrían ser el caso o que necesariamente deberían ser el caso, si se dieran tales o cuales circunstancias. Asumiendo el mosaico humeano amodal sobre el cual se erigen la teoría de la superveniencia humeana y la teoría del mejor sistema, ¿qué estatus modal pueden tener las leyes desde la perspectiva lewisiana? Revisaremos en detalle esta respuesta con el objetivo de dejar planteado el problema para los capítulos siguientes. 


\section{El desafío del humeanismo}

En la época de la emergencia de la investigación fisicomatemática de la naturaleza, Hume mostró plena conciencia de las diferencias que separaban, como un abismo, la tradición matematizante de la filosofía natural y la tradición especulativa de la filosofía moral y de la metafísica. La filosofía natural de los siglos XVII y XVIII se apropió de las metodologías experimentales y matemáticas de investigación, que en los siglos XIX y XX la conducirían a dar lugar a las ciencias físicas y matemáticas tal como las conocemos hoy. A tal filosofía natural Hume opone la filosofía moral, que dice relación con los asuntos humanos, y que incluye con límites borrosos la reflexión sobre la ética, la política, el arte, la historia y la metafísica, entre otras formas del saber. En Una Investigación sobre el Entendimiento Humano, Hume (2000 [1748]) sostiene que la gran ventaja de la filosofía natural matematizada por sobre la filosofía moral consiste en que las ideas de la primera son siempre claras y precisas, siendo una virtud de sus términos que ellos siempre expresen las mismas ideas sin ambigüedad ni variación. Entre tales términos Hume tiene en mente las figuras geométricas y las reglas de deducción de las matemáticas. Por el contrario, el gran obstáculo de las ciencias morales y metafísicas, en la expresión de Hume, es la obscuridad de sus ideas y la ambigüedad de sus términos, que la han llevado a progresar menos que la geometría o la física.

La dificultad en la clarificación y precisión de los conceptos es una de las razones que motivan la crítica a las tradiciones metafísicas precedentes. En un conocido pasaje, Hume afirma: "No hay ideas que ocurran en la metafísica que sean más obscuras e inciertas que aquellas de poder, fuerza, energía o conexión necesaria" (Hume 2000, 50. Mi traducción). La crítica humeana apunta directamente tanto a la ontología de poderes causales del aristotelismo escolástico como a la ontología de conexiones necesarias legaliformes del mecanicismo teológico de Descartes y Newton. Pues bien, la crítica de Hume se fundamenta en una epistemología característica del empirismo. El filósofo escocés sostiene que las fuentes de nuestro conocimiento imponen un límite para nuestro acceso a cuestiones ontológicas asociadas a poderes causales y conexiones necesarias. El argumento es conocido: nuestro conocimiento comienza con la experiencia sensorial que nos provee una serie de impresiones provocadas por los objetos que afectan a nuestros sentidos. La mente, a continuación, copiaría estas impresiones en ideas que conforman el material de trabajo con el que opera la imaginación. Y aunque el conocimiento comienza con las impresiones, no se restringe a ellas, puesto que la imaginación puede emplear su capacidad para asociar ideas simples en ideas complejas, obteniendo resultados tales como la idea de unicornio y de triángulo, que jamás se nos presentan a la experiencia sensorial, pero que se componen a partir de ideas más simples.

Piénsese entonces en la causalidad: cuando vemos que una primera bola de billar, C, causa el movimiento de una segunda bola de billar, E, lo que se manifiesta a nuestros sentidos es la relación de contigüidad espacial y de sucesión temporal entre C y E. Con ello a la vista, podemos articular dos grandes interpretaciones del alcance de la crítica humeana

Revista de Humanidades de Valparaíso, 2021, No 17, 145-167

(c) $(9)(9)$ CC BY-NC-ND 
a la metafísica que le precede. La primera consiste en una lectura ontológica, según la cual Hume negaría la realidad de los poderes causales y de las conexiones necesarias. El mobiliario del mundo estaría conformado exclusivamente por las entidades amodales de la ontología mecanicista, que instanciarían diversos patrones causales en el universo según su distribución espaciotemporal, sin descansar en los postulados metafísicos de poderes causales o conexiones necesarias. Algunos pasajes parecen motivar esta interpretación. Dice Hume (2000, 58. Mi traducción): "todos los eventos parecen estar completamente sueltos y separados [loose and separate]. Un evento sigue a otro; pero jamás podemos observar algún vínculo entre ellos. Ellos parecen estar en conjunción [conjoined], pero nunca conectados [connected]". Aunque el empirismo podría dar lugar a la interpretación ontológica, ello no estaría libre de problemas, principalmente si se considera que la lectura ontológica comete el error categorial de derivar consecuencias ontológicas a partir de cuestiones meramente epistémicas. La teoría de la actividad mental humeana, que incluye impresiones e ideas, además de la composición de ideas complejas a partir de ideas simples, caracteriza el alcance y límites generales de nuestro conocimiento, pero no determina cuestiones de existencia.

La segunda interpretación, en cambio, proviene de una lectura epistemológica, según la cual, al observar que $\mathrm{C}$ causa $\mathrm{E}$, las impresiones del poder causal o de la conexión necesaria permanecen siempre ocultas a nuestros sentidos. Dice Hume: "las escenas del universo están cambiando continuamente, y un objeto sigue a otro en una sucesión ininterrumpida; pero el poder o fuerza que actúa sobre la maquinaria completa permanece enteramente oculto para nosotros" (2000, 51. Mi traducción). De acuerdo con esto, el empirismo humeano no se aventura a sostener que no existen aquellas entidades que la tradición metafísica ha llamado poderes causales y conexiones necesarias. En cambio, la actitud que se adopta es agnóstica, sugiriendo que ignoramos si tales supuestos ontológicos existen en la medida en que escapan a los límites de nuestro conocimiento.

No queda claro si Hume distingue entre las interpretaciones epistemológicas y ontológicas en sus propios escritos. No obstante, la distinción nos permite preguntar con más precisión: ¿en qué consiste, pues, el establecimiento de relaciones causales del tipo $\mathrm{C} \rightarrow \mathrm{E}$ ? Tales relaciones representan regularidades en dos acepciones, a saber, ontológica y epistemológica. La primera nos ofrece una imagen de la realidad constituida exclusivamente por regularidades sin fundamento. Dada la ontología del mecanicismo, lo que hay en la realidad es el conjunto de entidades y sus propiedades que dan lugar a otros tantos procesos y eventos, todos ellos desprovistos de fuerza modal. Lo que observamos, desde la perspectiva de la investigación científica, es la emergencia (o aparición, sin más) de relaciones causales que tienen lugar en los diversos patrones de la naturaleza, determinados por la distribución espaciotemporal que las entidades y sus propiedades simplemente sucede que es el caso que instancian. Las escenas del universo se siguen unas a otras, dice Hume, y unos objetos parecen seguir a otros ininterrumpidamente, dando lugar a las regularidades que observamos. Aún más, desde la perspectiva empirista, la pregunta por la 
causa ulterior de las regularidades es ilegítima, puesto que tales fundamentos metafísicos escapan en principio a los límites de nuestras capacidades epistémicas y solo podrían ser objeto de especulación metafísica. Lo que hay son regularidades, conjunciones de unos objetos con otros, pero no conexiones fundamentadas en poderes causales o conexiones necesarias.

En cambio, la acepción epistémica de $\mathrm{C} \rightarrow \mathrm{E}$ se deriva de la teoría de la mente de Hume. Las impresiones ilustran cierta regularidad: pasamos de ver fuego a sentir el calor de este, o de sentir el calor a ver un trozo de papel quemarse; igualmente, pasamos de representarnos la idea de fuego a instanciar las ideas de calor o de un trozo de papel quemándose. Tras generar un hábito en las cadenas representacionales, la imaginación nos conduce al establecimiento de ciertas regularidades en nuestro pensamiento que sirven de reglas de inferencia para anticipar los fenómenos. En este caso, la regularidad es un hábito del pensamiento.

Las interpretaciones metafísicas y epistémicas de la relación causal $\mathrm{C} \rightarrow \mathrm{E}$ se confunden en la teoría humeana de la causalidad, para la cual Hume $(2000,60)$ ofrece tres definiciones:

i. Regularidad causal: tenemos un objeto seguido por otro, en donde todos los objetos similares al primero son seguidos por objetos similares al segundo;

ii. Causalidad contrafáctica: se instancia una relación entre dos objetos, en donde si no hubiera tenido lugar el primero, entonces tampoco habría tenido lugar el segundo;

iii. Causalidad psicológica: la relación causal es aquella en donde un objeto es seguido por otro, y la aparición del primero siempre conduce a la mente a la idea del segundo.

La tercera definición de causalidad acomoda a cabalidad la teoría de la mente de Hume. La regularidad causal tendría lugar en la sucesión de ideas originada por el establecimiento de hábitos del pensamiento, según la cual, tras instanciar la idea $\mathrm{C}$, la mente pasa por hábito a instanciar la idea E. Esta interpretación es interesante en el contexto de la teoría de la mente de Hume, pero no tanto así en su metafísica o filosofía de las ciencias, puesto que, en general, cuando en estas últimas nos preguntamos por la naturaleza de la causalidad, lo hacemos porque nos interesa elucidar el estatus ontológico de las relaciones causales entre los fenómenos y no entre nuestras ideas.

Las teorías causales de la regularidad y contrafáctica logran esto último desde perspectivas diferentes. La teoría de la regularidad es, por excelencia, la teoría de Hume, en tanto ella enuncia, en consonancia con numerosos pasajes de su obra, que la relación causal es aquella en donde un objeto es seguido por otro regularmente. Hume da un paso adicional al confiar en la epistemología de la inducción: vemos que $\mathrm{C}$ es seguido por $\mathrm{E}$ en 
un número de observaciones, y a partir de ello inducimos que todos los objetos similares a C serán seguidos por objetos similares a E. En este escenario, el análisis del significado de la semejanza podría traer consecuencias dispares. En una lectura minimalista, uno podría sostener que Hume se refiere a semejanzas en sus apariencias: los Cs y los Es parecen similares a nuestra percepción, y nosotros los clasificamos inductivamente como los mismos Cs y Es en sus apariencias, sin postular inobservables tales como las identidades de las sustancias aristotélicas. ${ }^{1}$ Una segunda interpretación, en cambio, sostendría que las semejanzas de los Cs y los Es descansa en que hay ciertas propiedades que ellos comparten, sirviendo de fundamento para las semejanzas que observamos. Pero esta última interpretación no se encuentra disponible para Hume si las propiedades se entienden como poderes causales, puesto que no tenemos acceso a estos últimos.

El enfoque contrafáctico ofrece, igualmente, regularidad y algo más. Si C no hubiera tenido lugar, entonces E no habría tenido lugar tampoco. ¿No es, acaso, ese el vínculo (tie) que necesitamos para establecer algo más que una mera conjunción entre $\mathrm{C}$ y $\mathrm{E}$ ? El análisis contrafáctico nos permite investigar escenarios experimentales en los que se observa la relación entre C y E. Si C no es el caso, entonces E no sería el caso. ¿De qué manera $\mathrm{C}$ es necesario para causar o producir E? En primer lugar, E podría todavía ser el caso aún cuando $\mathrm{C}$ no tuviera lugar, puesto que podríamos encontrar todavía una causa, $\mathrm{C} 1$, que produce $\mathrm{E}$. Los caminos de la naturaleza no son unívocos, y generalmente esta se las arregla para llegar a un resultado con medios diferentes. Este, no obstante, no es una estrategia argumentativa que encontremos en los escritos de Hume. ${ }^{2}$ En segundo lugar, asumiendo la relación contrafáctica entre $\mathrm{C}$ y E, cabe todavía mostrar que no necesariamente hemos encontrado el cemento de la relación causal entre ambas. ${ }^{3}$ Lo que observamos, a través de los lentes de la epistemología empirista, es que, si $\mathrm{C}$ es el caso, entonces E será el caso. Y observamos ello recurrentemente, sin que nuestras observaciones nos permitan inferir o descubrir que hay una propiedad causal en $\mathrm{C}$ que esté necesariamente conectada con alguna propiedad causal en E.

En este marco teórico, ¿qué puede decir Hume acerca de las leyes de la naturaleza? Las leyes de la naturaleza no ocupan un lugar central en la filosofía sistemática de Hume. En sus obras encontramos secciones acerca de causalidad, determinismo, libertad y milagros, entre otros, pero ninguna sección dedicada a la elaboración de una concepción de leyes de

\footnotetext{
${ }^{1}$ Agradezco a uno de las/os evaluadoras/es de la RHV por indicar lo siguiente: la inducción es una inferencia ampliativa cuyas premisas están constituidas por casos similares. La inducción misma no es la inferencia que permita identificar la similitud, sino que, en cambio, trabaja sobre ella. La presente observación se aplica sin problemas al caso del análisis humeano y conviene tenerlo en cuenta en el análisis causal.

${ }^{2}$ El problema ha sido abordado sistemáticamente con posterioridad. Véase Woodward (2003) y Lange (2000, 2009), entre otros.

${ }^{3}$ La expresión cemento refiere al análisis clásico de Mackie (1980) en su célebre The Cement of the Universe.
} 
la naturaleza que se posicione ante las ontologías del aristotelismo escolástico o el mecanicismo temprano de la filosofía natural. En algunos pasajes, Hume refiere a las leyes de Newton, pero sin ofrecer un análisis filosófico detallado.

Esto podría equívocamente indicar que el empirismo humeano no es tierra fértil para la reflexión sobre leyes de la naturaleza. Por el contrario, las ideas de Hume dan lugar a lo que se ha llamado teoría de la regularidad de las leyes de la naturaleza. ${ }^{4}$ Esta sostiene que, en términos ontológicos, las leyes de la naturaleza son regularidades instanciadas por patrones espaciotemporales fenoménicos; o en términos teóricos, que las leyes de la naturaleza son enunciados que expresan tales regularidades. Ello podría satisfacer las expectativas humeanas originales: todo lo que observamos son regularidades, sin postular ni tener acceso a los fundamentos metafísicos de las mismas. Entonces, si tenemos que hacernos cargo del hecho de que la práctica científica incluye leyes de la naturaleza, entonces nuestras opciones no son otras que sugerir que tales leyes son ontológicamente las regularidades que instancia el mundo, o que tales leyes son las que enuncian las regularidades observadas.

Nótese a continuación el problema infranqueable de la teoría de la regularidad que emerge desde una perspectiva metafísica: ella pareciera tornar superfluas las leyes de la naturaleza en la medida en que colapsa la distinción entre generalizaciones accidentales y generalizaciones nómicas. Estas últimas, para el humeanismo, no serían más que regularidades. ¿Por qué no limitarnos a hablar de regularidades, desechando la jerga de enunciados nómicos? Se necesitaría algo más que la mera regularidad para mantener en pie las leyes de la naturaleza. Pero, por lo pronto, el humeanismo ha cerrado las puertas para la especulación metafísica.

El desafío de Hume acerca de las leyes de la naturaleza ha resultado fructífero tanto en vistas de las concepciones cartesianas y newtonianas de las leyes (ver capítulo 2), como en vistas del debate reciente en filosofía de las leyes de la naturaleza. En vistas de lo primero, mientras que Descartes y Newton fundamentaron la necesidad de los movimientos de los cuerpos mecánicos en las leyes de la naturaleza, Hume propone una concepción de la causalidad que declina la postulación de la necesidad y se contenta con el establecimiento de regularidades a través de la observación. La propuesta de Hume es revolucionaria: si Descartes y Newton habían abandonado los poderes causales aristotélicos para reemplazarlos con las leyes de la naturaleza que gobiernan el universo mecánico, Hume nos insta a abandonar las leyes universales y necesarias que formularon Descartes y Newton para contemplar las regularidades causales en su desnudez modal.

\footnotetext{
${ }^{4}$ Véase, entre otros, Psillos (2002, capítulo 5). Psillos no adopta la teoría de la regularidad acerca de las leyes de la naturaleza, ni tampoco la teoría de la regularidad de la causalidad. Sin embargo, dada la claridad de sus exposiciones, ellas se han convertido en la exposición estándar de una visión que, hoy en día, no tiene un amplio respaldo en la literatura, a pesar de que se trata de un paso inevitable entre el mundo de particulares amodales de Hume y posteriores teorías de leyes de inspiración humeana.
}

Revista de Humanidades de Valparaíso, 2021, No 17, 145-167 
Ahora bien, más allá de su posicionamiento histórico, la publicación de las reflexiones humeanas sobre causalidad en el Tratado (Hume 1978 [1739]) y en la Investigación (Hume 2000 [1748]) traza una línea de investigación que será materia de discusión hasta nuestros días. De las ideas de Hume que hemos revisado hasta aquí se derivan estrategias para abordar los siguientes tres asuntos:

i. Leyes: teoría y ontología. En las secciones siguientes argumentaremos que desde la perspectiva humeana las leyes son enunciados, a saber, teorías, que expresan generalizaciones empíricas acerca de regularidades. La discusión tradicional, sobre todo en la tradición metafísica, no se sentiría satisfecha con esto, sino que propondría que las leyes de la naturaleza forman parte del inventario ontológico de la realidad.

ii. Modalidad: de re y de dicto. Esta segunda distinción es similar a la primera, pero se concentra en nuestra concepción de la necesidad. Asumiendo que las leyes de la naturaleza debieran ser eminentemente modales. Mientras que la tradición metafísica sostiene que la necesidad es una propiedad modal de aquellos fenómenos que ostentan un estatus nomológico genuino, las teorías humeanas sostendrán que la necesidad es un predicado del lenguaje natural o formal con el que formulamos nuestras teorías. Las regularidades amodales del mundo solamente nos informan contingencias.

iii. Dirección de la explicación: top down y bottom up. Ya antes hemos introducido la distinción entre estas dos estrategias explicativas. La concepción metafísica sostiene que las leyes de la naturaleza gobiernan los fenómenos de manera top down, vale decir, como si ellas representaran un estrato ontológico superior que determina lo que necesariamente debe ser el caso en los fenómenos. La concepción humeana, en cambio, adopta la estrategia bottom up, en tanto que, si hay leyes de la naturaleza, ellas constituirían el resultado de generalizaciones empíricas a partir de las regularidades que observamos en los fenómenos. Serían, en consecuencia, estas regularidades las responsables de las generalizaciones empíricas que pretendemos etiquetar como leyes.

\section{Superveniencia humeana y mejor sistema}

En esta sección y en las siguientes examinaremos en detalle el humeanismo nomológico contemporáneo con sus consecuencias para el estatus de las leyes, la modalidad y la dirección de explicación. Revisaremos la elaboración y defensa de la teoría de la superveniencia humeana y la teoría del mejor sistema, que encontramos en los trabajos de David Lewis. Este último, de hecho, ha revitalizado exitosamente el proyecto nomológico humeano en metafísica contemporánea. 


\subsection{Superveniencia humeana}

La superveniencia humeana lleva su nombre en honor al gran negador de las conexiones necesarias en la naturaleza. Esta tesis sostiene que todo lo que existe en la realidad es solamente un basto mosaico de cuestiones locales de hechos particulares. Tal mosaico se representa en términos de relaciones externas de distancias espaciotemporales entre puntos -o cosas que, para propósitos del argumento, tenemos que imaginarnos como si ocuparan un punto en el espaciotiempo. Según ello, lo que existe es el arreglo espaciotemporal de cualidades locales, a saber, propiedades intrínsecas perfectamente naturales a partir de las cuales superviene el resto. Lewis presenta su propuesta así:

[La superveniencia humeana] dice que en un mundo como el nuestro las relaciones fundamentales son exactamente relaciones espaciotemporales: relaciones de distancia, tanto espaciales como temporales, y quizás también relaciones de ocupación entre cosas que tienen el tamaño de un punto y puntos espaciotemporales. Y esta dice que, en un mundo como el nuestro, las propiedades fundamentales son cualidades locales: propiedades intrínsecas perfectamente naturales de puntos, o de ocupantes de puntos del tamaño de un punto. Por consiguiente, esta dice que todo lo demás superviene del arreglo espaciotemporal de cualidades locales a través de la historia pasada, presente y futura. (Lewis 1994, 474. Mi traducción)

Las propiedades intrínsecas perfectamente naturales (así como las relaciones perfectamente naturales) son aquellas que constituyen el patrón de instanciación que da lugar a la estructura fundamental de la realidad. Algunos ejemplos de propiedades intrínsecas perfectamente naturales son la carga eléctrica, la masa y el spin de un electrón, que determinan las relaciones causales de las que participa esta entidad. En su calidad de anti-haecceitista, la tesis de la superveniencia humeana sostiene que todo superviene del solo patrón de coinstanciación de propiedades, vale decir, de las relaciones espaciotemporales que ellas instancian, sin importar la elucidación de las sustancias a las que pertenecen. Lewis $(1994,474)$ manifiesta su confianza con respecto a la posibilidad de que en algún momento la física nos ofrezca un inventario completo de las propiedades y relaciones perfectamente naturales, tales como la masa, carga eléctrica y spin de los electrones, permitiéndonos explicar lo que superviene a partir de ellas.

El espíritu de la propuesta de Lewis es declaradamente humeano: concebimos el universo compuesto de propiedades y relaciones amodales, que se encuentran distribuidas en el espaciotiempo en términos de relaciones espaciotemporales. Desde la perspectiva de la investigación empírica, nosotros observamos este patrón de instanciación, la llamada regularidad humeana, sin nunca elucidar putativos cimientos modales que fundamenten las relaciones espaciotemporales en términos de poderes causales o conexiones necesarias. 


\subsection{Teoría del mejor sistema}

Recuérdese el desafío de Hume respecto a las leyes de la naturaleza: si hay leyes, tales leyes no podrían ser sino regularidades o enunciados de regularidades. Al despojar a la realidad física de cualquier sustrato modal, que la ontología precedente obtenía a partir de los poderes causales aristotélicos y de las conexiones necesarias que Descartes y Newton obtenían de Dios, Hume nos ofrece una descripción de la realidad que solamente incluye regularidades contingentes. El desafío de Hume cobró cada vez más importancia en la medida en que las leyes físicas proliferaron durante los siglos XVIII, XIX y XX. ¿Cómo podría el programa humeano ofrecer una teoría acerca de las leyes de la naturaleza, que dé cuenta de las leyes que encontramos en la práctica científica? Lewis responde a esta pregunta afirmando que una regularidad es una ley si y solo si es un teorema en el mejor sistema:

Considérense todos los sistemas deductivos cuyos teoremas son verdaderos. Algunos son más simples y están mejor sistematizados que otros. Algunos son más fuertes, más informativos, que otros. Estas virtudes compiten: un sistema no informativo puede ser muy simple, y un compendio no sistematizado de información miscelánea puede ser muy informativo. El mejor sistema es aquél que logra un balance tan bueno entre la simplicidad y la fuerza como el que permita la verdad. Cuán bueno sea el balance dependerá de cuán amable sea la naturaleza. Una regularidad es una ley si y solo si es un teorema del mejor sistema. (Lewis 1994, 478. Mi traducción)

La imagen que ofrece Lewis es atractiva: dado el mosaico humeano de la tesis de la superveniencia, nuestro constructo teórico acerca de la realidad contiene teorías que enuncian generalizaciones empíricas acerca del patrón de coinstanciación de propiedades y relaciones perfectamente naturales. Nuestras teorías acerca del mundo describen, entre otras cosas, relaciones espaciotemporales entre particulares. Por supuesto, nuestro constructo teórico total incluye teorías cuyo alcance empírico es diverso. Algunas proposiciones se limitan a enunciar relaciones estrictamente limitadas entre algunos fenómenos en términos espaciotemporales, como cuando profiero la proposición mi taza de café está sobre el escritorio. Una teoría que expresa una generalización empírica de mayor alcance, en cambio, sería la teoría molecular del ADN, que se aplica a organismos vivos sobre la faz de la tierra. Y otra teoría cuya generalización empírica tiene todavía un alcance mayor es el postulado de la velocidad de la luz, que nos dice que nada en el universo puede moverse más rápido que la velocidad de la luz. Desde la perspectiva de la teoría del mejor sistema, las leyes de la naturaleza son aquellas proposiciones que logran un mejor balance entre simplicidad y robustez, adecuándose así de mejor manera a lo que observamos en la práctica científica. 
¿Cómo se entienden los criterios de simplicidad y robustez? Primero, la simplicidad descansa en la estructura sintáctica de los enunciados teóricos, y en el número de postulados ontológicos que ellos introducen. Y segundo, la robustez refiere, por cierto, a la información que ofrece un enunciado cualquiera, particularmente en vistas de los posibles patrones de coinstanciación del mosaico humeano que prohíbe. El mejor balance que pueda lograrse entre ambos criterios en un enunciado determina si es el caso que una de nuestras teorías puede, efectivamente, ocupar el lugar de un teorema en un sistema axiomático, constituyendo una ley. Considérense los enunciados antes mencionados. La proposición mi taza de café está sobre la mesa del escritorio ofrece información estrictamente restringida al contexto de uso, y aunque postula una ontología aparentemente mínima, ella no ofrece información científicamente relevante acerca de un patrón de coinstanciación del mundo físico. En cambio, la teoría molecular del ADN, que introduce postulados ontológicos acerca de la estructura molecular del ácido desoxirribonucleico, la composicionalidad genética y la información, nos ofrece una generalización empírica ampliamente informativa acerca del desarrollo y reproducción de los organismos. Se negocia entre la simplicidad sintáctica del enunciado y de sus postulados ontológicos, por un lado, y el poder informativo de la generalización ofrecida, por otro. La negociación, por cierto, debe salvaguardar la informatividad de la teoría relevante en cada caso en vistas de una serie de consideraciones tales como su contribución a la derivación de inferencias, su relevancia dentro del sistema teórico y su indispensabilidad epistémica, entre otras. Considérese el caso del segundo postulado de la relatividad especial sobre la velocidad de la luz, que dice que nada en el universo puede desplazarse más rápido que la velocidad de la luz dada por la constante $\mathrm{C}$, vale decir, aproximadamente $3.00 \times 10^{8} \mathrm{~m} / \mathrm{s}$ en el vacío. Este postulado, por un lado, combina la simplicidad sintáctica y robustez informativa acerca de un dominio de la realidad, postulando la constante de la velocidad de la luz como cantidad física y ofreciéndonos amplia información física acerca de escenarios de patrones de coinstanciación que prohíbe, a saber: que nada, sea cual fuere el particular al que nos refiramos, pueda moverse más rápido que la velocidad de la luz.

El siguiente cuadro esquematiza las ideas centrales de la tesis de la superveniencia humeana y la teoría del mejor sistema: 


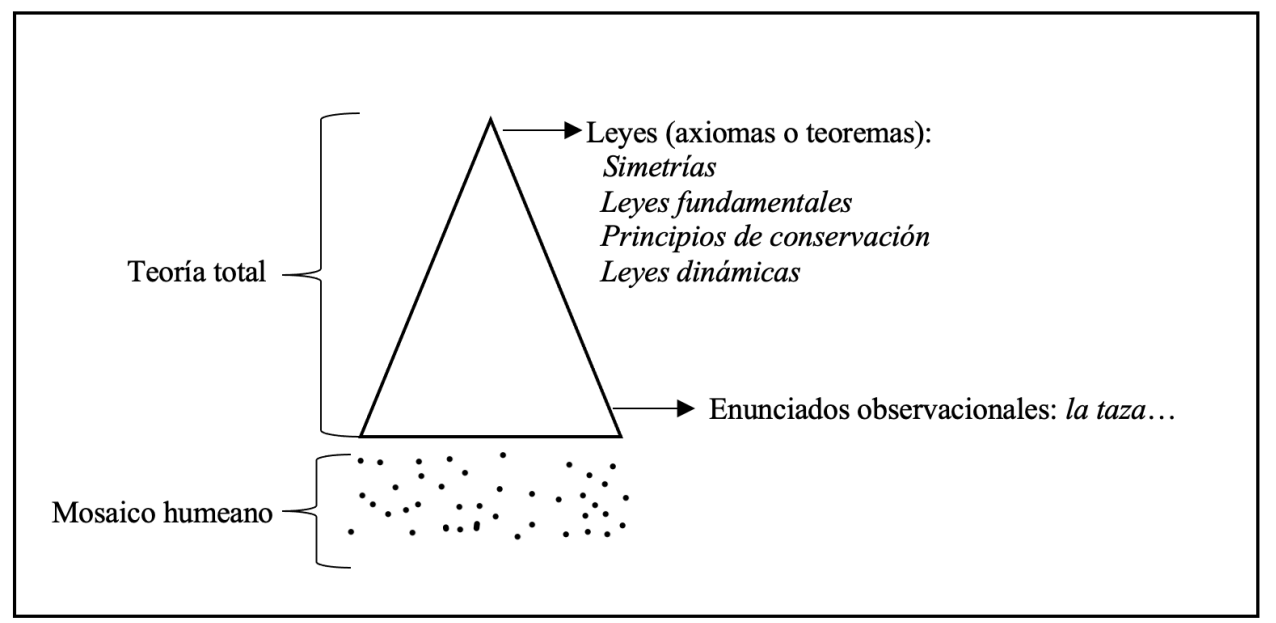

Las virtudes de la teoría del mejor sistema, en conjunto con la tesis de la superveniencia humeana, son diversas. Primero, la propuesta lewisiana nos permite escapar al universo sobrepoblado. Si lo que se quiere es parsimonia ontológica, entonces la ontología de la superveniencia humeana y del mejor sistema ofrece una buena receta. La realidad está últimamente constituida de propiedades intrínsecas perfectamente naturales y las relaciones espaciotemporales que se instancian entre ellas. No aparecen, en su fundamento, propiedades modales de ningún tipo, y los patrones de coinstanciación dan lugar a las regularidades que describimos con las leyes de la naturaleza que encontramos en la práctica científica. Tales leyes enuncian, de manera simple e informativa, las mejores generalizaciones formuladas en la investigación. La propuesta lewisiana mantiene en pie la ontología humeana deflacionaria, aunque, como veremos, no sin consecuencias.

Una segunda virtud es esta: la teoría del mejor sistema preserva dos intuiciones ampliamente compartidas en diversas interpretaciones de la práctica científica. La primera sostiene que las leyes de la naturaleza ocupan el lugar de principios (Lewis habla de axiomas o teoremas) en un sistema teórico deductivo, permitiéndonos inferir proposiciones de menor alcance que explican o predicen los fenómenos relevantes. La segunda intuición dice relación con el reduccionismo, puesto que los enunciados de leyes pueden ser reducidos a los arreglos espaciotemporales de distribución de propiedades y relaciones perfectamente naturales. Las leyes no introducen espíritus metafísicos fantasmagóricos, sino que ellas se reducen a la constitución del mosaico humeano.

\section{Problemas y desarrollos recientes}

Revisemos a continuación algunos de los problemas que enfrenta la propuesta lewisiana, tanto en vistas de la tesis de la superveniencia humeana como en vistas de la teoría del mejor sistema. La literatura reciente se ha encargado de examinar estos problemas 
sistemáticamente, ofreciendo en ocasiones adiciones a la teoría del mejor sistema que permitirían subsanar al menos algunas de las dificultades. El debate ha crecido exponencialmente. Desde los análisis de la superveniencia humeana por parte de Loewer (2004) y la defensa exhaustiva de la superveniencia humeana en las contribuciones de Earman y Roberts (2005a, 2005b), la producción bibliográfica le ha asegurado a la propuesta lewsiana un lugar propio en el debate reciente. Se ha defendido una versión mejorada del mejor sistema (better best system, Cohen y Callender 2009), expandiendo la propuesta de Lewis más allá de la física hacia las ciencias especiales; otros han articulado lo que sería el mejor sistema para nosotros (best system for us, Jaag y Loew 2018), destacando la optimización cognitiva y pragmática de los enunciados de leyes; e incluso se han propuesto aproximaciones perspectivales a la versión mejorada del mejor sistema (perspectivalist better best system, Massimi 2018), interpretaciones que destacan la robustez predictiva del mejor sistema (predictive best system, Dorst 2019), e interpretaciones que resaltan el modelamiento metafísico de la tesis de la superveniencia humeana en conjunto con la teoría del mejor sistema (Jaag y Loew 2020). En general, la literatura coincide en que ciertos problemas aparecen recurrentemente. Examinemos tres de ellos a continuación.

\section{1. ¿Encuentra la propuesta lewisiana fundamento en la física?}

Según el mismo Lewis, la tesis de la superveniencia humeana está inspirada en la física clásica. Se nos habla de sistemas axiomáticos similares a la geometría de Euclides; se nos habla de relaciones espaciotemporales de distancia entre cosas que tienen el tamaño de un punto; y se nos dice que las leyes de la naturaleza son los axiomas del sistema deductivo. Sin embargo, ello no parece adecuarse a lo que nuestra física fundamental actual nos enseña en mecánica cuántica. Ante ello, hay quienes sostienen: "parece altamente improbable que el mundo de Lewis de 'propiedades intrínsecas perfectamente naturales de puntos, o de ocupantes de puntos del tamaño de un punto' sea el mundo actual" (Ladyman et al. 2007, 19-20. Mi traducción). El mundo lewisiano sería pseudocientífico: nuestra mejor teoría fundamental indica que no vivimos en un mundo newtoniano.

Si le exigiéramos a la teoría de Lewis que dé un paso adelante en la historia y que se haga cargo de, digamos, la mecánica cuántica, entonces enfrentaría problemas irreversibles. La mecánica cuántica no garantiza que las propiedades fundamentales que ella postula sean propiedades intrínsecas perfectamente naturales, localizadas sin problema en cada una de sus instanciaciones. Loewer, entre los más connotados defensores de la superveniencia humeana, es claro al respecto:

parece sumamente claro que la física contemporánea sueña con propiedades no humeanas. Tengo en mente los llamados estados de entrelazamiento que son responsables de la no-localidad cuántica, i.e., de las violaciones de las desigualdades de Bell en mecánica cuántica. Los estados de entrelazamiento de un par de partículas no 
supervienen de las propiedades intrínsecas de las partículas separadas. Esto es, las propiedades locales de las partículas no determinan separadamente el estado cuántico completo y no determinan específicamente cómo se vinculan las evoluciones de las partículas. (Loewer 2004, 179. Mi traducción)

La teoría de propiedades de Lewis, con su requerimiento de localidad e instanciación, no permite explicar las violaciones de teorema de Bell. Las propiedades que posee un estado de entrelazamiento no supervienen de la sola adición de las propiedades iniciales de las dos partículas relevantes tomadas por separado.

La respuesta de Lewis ante este problema es inquietante, pues dice no estar "preparado para desprender lecciones en ontología a partir de la física cuántica tal como está ahora [viz., 1986]. Primero tengo que ver cómo luce cuando sea purificada de frivolidad instrumentalista" (Lewis 1986, xi. Mi traducción). Lewsianos contemporáneos, en cambio, han ido más lejos en la respuesta. Si bien cabe reconocer que la superveniencia humeana y el mejor sistema están inspirados en mecánica clásica y que carecen de herramientas para acomodar la mecánica cuántica, la propuesta de Lewis tiene que ser evaluada en tanto modelo metafísico y no en tanto descripción literal de la ontología de la física fundamental (Jaag y Loew 2020). Desde esta perspectiva, Lewis debate con otras propuestas metafísicas: "el punto con defender la superveniencia humeana no tiene que ver con apoyar la física reaccionaria, sino más bien con resistir los argumentos filosóficos que dicen que hay más cosas en el cielo y en la tierra que aquellas que la física podría soñar" (Lewis 1994, 474. Mi traducción). Según esto, si lo que nos interesa es ofrecer una explicación de las leyes de la naturaleza, bastaría con la tesis de la superveniencia humeana y la teoría del mejor sistema nos provean una caracterización competitiva de las leyes de la naturaleza y de su rol en la praxis científica, permaneciendo fiel al minimalismo humeano. La propuesta de Lewis podría lograr eso, consolidándose como un modelo metafísico que busca dar cuenta del lenguaje modal de las leyes científicas.

\subsection{Circularidad explicativa}

La propuesta lewsiana sostiene que el mosaico humeano es un fundamento ontológico indispensable que permite explicar las leyes de la naturaleza que incluye nuestro mejor sistema. Ahora bien, serían esas mismas leyes las que en la práctica científica nos permitirían explicar los patrones estructurales del mosaico humeano. Surge la pregunta: ¿qué explica qué? ¿El mosaico humeano a las leyes? ¿O las leyes al mosaico humeano? Una definición clara de explanans (lo que explica) y explanandum (lo explicado) resulta necesaria.

Usualmente se asume en la discusión sobre leyes de la naturaleza que son estas las que permiten explicar los fenómenos. En su versión más fuerte, la tesis de que las leyes explican los fenómenos que caen bajo su dominio toma la forma de la concepción no- 
mológica gobernante (governing view), según la cual las leyes gobiernan las relaciones causales entre fenómenos. La metáfora del gobierno, sin embargo, no resulta cómoda hoy en día, considerando su carácter teológico en la filosofía natural, en donde el gobernador en última instancia es el Dios del judeocristianismo que impone las leyes sobre el mundo mecánico.

La situación con la propuesta de Lewis es diferente: la teoría del mejor sistema depende de la superveniencia humeana cuyo punto de partida es el mosaico humeano amodal. ${ }^{5}$ Nuestras teorías, según Lewis, refieren a patrones estructurales, instanciados por la distribución espaciotemporal de propiedades intrínsecas perfectamente naturales y sus relaciones. Aquellas teorías que se encuentren entre las mejores que tenemos optarán a ser jerarquizadas en calidad de axiomas o principios en el mejor sistema deductivo. Sin embargo, quienes defienden la tesis de la superveniencia humeana podrían todavía sostener que la teoría del mejor sistema no depende exclusivamente del mosaico humeano, sino que se requiere de nuestra intervención al calibrar el mejor balance entre simplicidad y robustez informacional de las teorías. Solamente aquellas que logren el mejor balance ostentarán el estatus de leyes de la naturaleza. En ello la superveniencia humeana tiene un punto a su favor: las leyes de la naturaleza no emergen sin más del mosaico humeano, sino que la comunidad epistémica relevante trabaja en aras a obtener el mejor balance entre simplicidad y robustez informacional.

Ello, no obstante, no resuelve el problema de la dirección de la explicación: las teorías que lleguen a ocupar el lugar de leyes en nuestro sistema deductivo serán aquellas que satisfagan los criterios de simplicidad y robustez informacional, pero aún así emergerán a partir de los patrones de instanciación de propiedades y relaciones perfectamente naturales. El mosaico humeano seguiría siendo parte indispensable de la explicación de las leyes que tenemos, sin lograr escapar de la amenaza de la circularidad: ¿cómo podrían las leyes de la naturaleza explicar los fenómenos del mosaico humeano si, a la vez, este mosaico humeano (junto a los criterios de simplicidad y robustez informacional) es un fundamento indispensable para explicar la emergencia de las leyes de la naturaleza? Se mantiene en pie el enredo conceptual entre explanans y explanandum.

\footnotetext{
${ }^{5}$ Nótese que la metafísica lewisiana tiene al menos dos partes: una, que es aquella con la que lidiamos en este artículo, dice relación con la ontología humeana amodal que remite a la distribución espacio-temporal de propiedades locales y relaciones; la otra parte de la metafísica lewisiana es, por el contrario, profundamente modal, en tanto que recurre a la postulación de mundos posibles que se estiman concretos o al menos tan reales como este mundo. Consideraciones acerca de esta segunda parte de la metafísica lewsiana solo tangentemente tocan el desarrollo de nuestro argumento. Agradezco a uno/a de los/as evaluadores/as de la RHV por señalar esta observación.
}

Revista de Humanidades de Valparaíso, 2021, No 17, 145-167 


\subsection{Antropomorfismo}

Lewis sostiene: "El peor problema acerca del análisis del mejor sistema es que cuando preguntamos de dónde provienen los estándares de simplicidad y fuerza, la respuesta parecería ser que ellos provienen de nosotros" (Lewis 1994, 479. Mi traducción). El problema se hace patente cuando reparamos en que simplicidad y robustez informacional dependen de lo que las comunidades epistémicas relevantes encuentren suficientemente simple y suficientemente robusto en sus disciplinas. Lewis no se inquieta demasiado con el problema del antropomorfismo. Sugiere, en pocas palabras, la siguiente respuesta:

si la naturaleza es amable con nosotros, el problema no tiene por qué surgir. Supongo que nuestros estándares de simplicidad y robustez y balance son solamente en parte una cuestión de psicología. No se debe a cómo sucede que nosotros pensamos que una función lineal sea más simple que una función cuadrática o de paso; no se debe a cómo sucede que nosotros pensamos que una alternación más corta de cuantificadores de prenex sean más simples que uno largo; y así sucesivamente. [...] Si la naturaleza es amable, el mejor sistema será el mejor robustamente. (Lewis 1994, 479. Mi traducción).

La respuesta depende entonces de la amabilidad de la naturaleza. En el mismo pasaje, Lewis sugiere que no tenemos razones para pensar que la naturaleza no sea amable con nosotros. Claramente estas dos afirmaciones no expresan argumentos de peso que debamos tener en consideración. Sin más, la respuesta lewisiana al problema del antropomorfismo de los criterios de simplicidad y robustez informacional es insuficiente. Piénsese en el lenguaje que empleamos. En tanto agentes epistémicos, el lenguaje ordinario resulta ampliamente simple para describir escenas del día a día; pero cuando nos adentramos en las teorías de las ciencias físicas, la situación cambia y recurrimos al lenguaje formal de las matemáticas. Este último tiene diversos grados de complejidad. Lewis menciona las funciones lineales y las cuadráticas, pero pareciera ser que las evalúa considerando exclusivamente la complejidad sintáctica de cada una de ellas. La complejidad de estas tiene que evaluarse teniendo en cuenta la información que nos permiten manipular en su aplicación a casos concretos. Ellas pueden ser altamente complejas en su sintaxis, pero aún así pueden constituir nuestro medio más simple disponible para modelar datos sobre aspectos de sistemas cuya complejidad ontológica nos resultaría intratable de otra manera. La simplicidad se negocia con la robustez: la sintaxis de una ecuación diferencial que expresa una ley de la naturaleza reviste potencial inferencial en vistas del sinnúmero de fenómenos que puedan caer bajo su alcance. En esta encrucijada, tanto la simplicidad como la robustez informacional dependen de la disponibilidad de teorías matemáticas adecuadas, así como de la cantidad de información que las comunidades epistémicas relevantes consideren suficiente para modelar un fenómeno particular. 
El antropomorfismo de nuestras concepciones no debiera causarnos temor. Después de todo, somos la clase de animales que tiene un conjunto finito de mecanismos cognitivos en un momento determinado, y no podemos sino trabajar con ellos y refinarlos en la medida en que podamos. Es inevitable, según esto, que ciertos aspectos de nuestra humanidad se plasmen en la manera en la que vemos el mundo. Las leyes de la naturaleza nos ofrecen información relevante para agentes cognitivos como nosotros, y en ellas encapsulamos la información física que nos es útil para diversos propósitos, entre ellos la explicación y predicción de eventos a partir de nuestra teorización acerca de eventos pasados (Jaag y Loew 2018). Los estándares de simplicidad y robustez informativa, junto al balance entre ellas, han evolucionado de manera notoria a través de la historia, dependiendo de asuntos tales como la disponibilidad de observaciones y mediciones adecuadas, y disponibilidad de modelos matemáticos óptimos. Simplicidad y robustez informativa, así como el mejor balance entre ellos, son determinados por las perspectivas que imponen las comunidades epistémicas en distintos momentos de la historia de la investigación de la naturaleza (Massimi 2018). La amenaza del antropomorfismo podría, según esto, no ser amenaza alguna, sino en cambio una variable más que tenemos que controlar en tanto que introduce la perspectiva que determina los grados óptimos de simplicidad y robustez informacional para nuestras leyes de la naturaleza.

\section{5. ¿Y la modalidad de las leyes?}

La modalidad es uno de los elementos claves para evaluar la propuesta humeana de Lewis. La tradición ha asociado a las leyes de la naturaleza con su estatus modal. El conjunto de las leyes de la naturaleza refiere a cuestiones que necesariamente son el caso, o que necesaria o posiblemente serían el caso si dieran tales y cuales condiciones, en oposición a aquellas cuestiones que suceden solo contingentemente. Además, las leyes de la naturaleza no se limitan a describir fenómenos actuales y pasados, sino que ellas determinan el espacio de posibilidad para el curso futuro de los eventos. Se espera que toda teoría de leyes de la naturaleza satisfaga este desiderátum, dando cuenta de la modalidad que hace que las leyes sean adecuadas para informarnos acerca de mucho más que la sola actualidad.

Las respuestas al problema de la modalidad en filosofía de leyes de la naturaleza son diversas. Permitámonos distinguir entre dos tipos de modalidad, a saber, modalidad de $r e$, que es modalidad realmente existente en la naturaleza; y modalidad de dicto, que es la modalidad que nosotros introducimos en el lenguaje que empleamos para referirnos al mundo. Descartes y Newton sostienen que la necesidad de las leyes de la naturaleza se fundamenta en la inmutabilidad de Dios, quien, a través de la inmutabilidad de su voluntad, determina que ciertos fenómenos sean necesariamente el caso. Construcciones metafísicas de las leyes de la naturaleza seguirán una ruta diferente, cuya orientación general es compartida a pesar de que presentan diferencias en los detalles: la fuente de modalidad 
de las leyes de la naturaleza descansa en diversas clases de entidades metafísicas, sean universales o esencias disposicionales, que nos permiten explicar la necesidad de las leyes o el hecho de que ellas refieran no solo a la actualidad sino también a la posibilidad. Estas propuestas sostienen, aunque a partir de motivaciones distintas, que la modalidad de las leyes de la naturaleza es eminentemente de re.

Nos preocupa entonces es saber si la concepción humeana de leyes, elaborada por Lewis, puede responder a la pregunta por la modalidad de las leyes de la naturaleza. La primera consideración la sugiere el mosaico humeano: éste está constituido por propiedades intrínsecas perfectamente naturales y las relaciones espaciotemporales que ellas instancian, sin encontrar rastros de modalidad en ninguna de sus partes. Y si el mosaico humeano es todo lo que hay en la naturaleza, y éste basta para explicar todo lo que le superviene, entonces una primera conjetura es la siguiente: la teoría lewisiana de leyes de la naturaleza falla a la hora de dar cuenta de la modalidad de re de las leyes de la naturaleza.

Se podría todavía argumentar que la modalidad de re es una propiedad sui generis que superviene al mosaico humeano. Si bien no encontramos modalidad de re en el mosaico humeano, podríamos encontrar modalidad de re en aquellos patrones instanciados por las relaciones espaciotemporales entre propiedades, que son los que describen nuestras leyes. La superveniencia de la modalidad de re, en este sentido, es problemática si se la mira desde la perspectiva humeana que inspira a la teoría del mejor sistema, puesto que en algún eslabón de la superveniencia tendría que emerger esta nueva propiedad, a saber, modalidad de re. Ello violaría el reduccionismo propio de la superveniencia humeana: si lo que superviene no es explicable en términos de las propiedades locales (instanciadas) del mosaico humeano, entonces ello no cuenta con las credenciales adecuadas para su existencia. La modalidad de re supervendría, pues, mágicamente.

La teoría del mejor sistema cuenta todavía con la modalidad de dicto: las leyes de la naturaleza son enunciados que describen patrones instanciados por las relaciones espaciotemporales entre propiedades del mosaico humeano, y las consideramos necesarias e incluso universales en la medida en que ellas forman parte, como axiomas o principios, del sistema teórico que obtenemos del mejor balance entre simplicidad y robustez informacional. En tanto enunciados, las leyes poseen modalidad de dicto: decimos que los enunciados de leyes son necesarios en tanto que ocupan el lugar de axiomas del sistema teórico, pero no decimos que los patrones del mosaico humeano que ellas describen sean modales, ni que la modalidad sea una propiedad mágica que emerja en alguno de los eslabones ontológicos de superveniencia.

Aunque Lewis es un realista modal acerca de los mundos posibles, ello no nos permite aclarar la cuestión de la modalidad de las leyes de la naturaleza. El realismo modal sostiene que todos los mundos posibles son reales al igual que nuestro mundo actual, en donde cualquier mundo posible podría ser el actual dependiendo del agente que emplee el indexical este mundo, que haga referencia a un aquí y ahora donde se encuentre. En

Revista de Humanidades de Valparaíso, 2021, No 17, 145-167 
este contexto, las leyes de la naturaleza podrían ser el conjunto de proposiciones que son verdaderas de todos los mundos posibles que comparten el mismo mosaico humeano. Y como todos los mundos posibles son de la misma clase (kind) que el nuestro, entonces ellos compartirían las mismas leyes, asumiendo que las comunidades epistémicas de los otros mundos posibles emplean los mismos criterios de simplicidad y robustez informacional - y que tienen el mismo grado de tolerancia acerca de determinados umbrales de simplicidad y robustez informacional. ${ }^{6}$ Pues bien, aún si asumimos que todo ello es así, seguiría siendo el caso que la modalidad de re no tiene lugar en el mosaico humeano (de cualquier mundo posible) y que esta solo se predica de las leyes de la naturaleza que enunciamos en las teorías que ocupan el lugar de axiomas en el mejor sistema.

Que la modalidad de las leyes de la naturaleza (su capacidad para enunciar necesidad y posibilidad) sea limitada a la modalidad de dicto no tiene que representar un problema sin más. Si bien no responde al desiderátum de las teorías estándares de leyes, que sostienen que la modalidad nomológica es de re, todavía podría defenderse que tal desiderátum carece de justificación. La modalidad de dicto se ajusta al espíritu humeano de la superveniencia humeana y del mejor sistema: lo que observamos son las regularidades o patrones de coinstanciación del mosaico humeano que es intrínsecamente amodal. Los poderes causales y las conexiones necesarias permanecen ocultas para nosotros. Solo vemos una cosa y luego otra, conjunción y no conexión, regularidad y no necesidad. La necesidad aparece, entonces, en la mente humana, cuando esta siente la inclinación a pensar en el efecto una vez que ha pensado en la causa. Pero no hay más que descubrir en ello: nuestras teorías pueden proyectar sobre el mundo la necesidad que formulamos en nuestras ideas y que expresamos con nuestro lenguaje natural o formal de la lógica o las matemáticas (Soto y Bueno 2019; Soto 2020a).

Aunque la aproximación humeana puede darse por satisfecha con la modalidad de dicto, cabe todavía preguntar por qué ciertos patrones que instancia el mosaico humeano llegan a ser el referente de aquellos enunciados que llamamos leyes de la naturaleza, mientras que otros patrones del mosaico humeano son referidos por teorías menores, que no llamaríamos leyes o principios. El mosaico humeano incluye por igual el segundo postulado de la relatividad especial acerca de la velocidad de la luz y el patrón de instanciación de esta taza que está sobre esta mesa en mi escritorio. Dada la simplicidad y robustez informacional del primer patrón, decimos que este corresponde a una ley y que es necesaria, pero usualmente no creemos que sea necesaria simplemente por una cuestión lingüística, sino porque la constitución del mundo físico hace que sea necesario que nada

\footnotetext{
${ }^{6}$ Según Lewis, si todos los mundos son actuales y están aislados entre sí, entonces un individuo en uno de estos mundos no sabría acerca de las regularidades de otros mundos, siendo imposible constatar la coincidencia en el conjunto de enunciados nómicos relevantes. Agradezco a una/o de las/os evaluadoras/es por la observación.
}

Revista de Humanidades de Valparaíso, 2021, No 17, 145-167

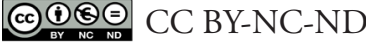


pueda viajar más rápido que la velocidad que puede alcanzar un fotón en el vacío. Pareciera, pues, haber una diferencia ontológica y no solo lingüística entre el primer patrón y el segundo. De ello no da cuenta la teoría del mejor sistema. ${ }^{7}$

\section{Conclusión}

En este artículo hemos examinado el humeanismo nomológico tanto en vistas de la teoría de la regularidad de Hume con en vistas de la teoría del mejor sistema (y su teoría aliada de la superveniencia humeana) de Lewis. Se trata de una línea fértil de reflexión sobre los problemas ontológicos y epistemológicos que suscita la adición de leyes de la naturaleza a nuestra concepción de mundo. Como tal, sin embargo, no corre libre de problemas, siendo aquellos de la adecuación con las teorías físicas, la circularidad explicativa y el antropomorfismo algunos de los que han recibido mayor atención en la literatura. El humeanismo nomológico deja, además, otro cabo suelto: no cuenta con las herramientas para ofrecer una concepción robusta de la modalidad usualmente asociada a las leyes físicas. En su rechazo de concepciones metafísicas de la modalidad, el humeanismo nomológico solamente puede recurrir a una concepción débil de la modalidad de dicto de enunciados de leyes. Nótese lo siguiente: desarrollos recientes del empirismo han comenzado a explorar otras posibilidades que puedan incluir una noción de modalidad física que cumpla con el minimalismo ontológico del empirismo y que, a la vez, salve nuestras intuiciones acerca de las leyes físicas (Soto y Rodríguez 2019; Soto 2020b). Tal línea de investigación se encuentra abierta al presente, y queda todavía por ver si logra dar con resultados satisfactorios.

\section{Referencias bibliográficas}

Cohen, J., Callender, C. (2009). A Better Best System Account of Lawhood. Philosophical Studies, 145(1), 1-34. https://doi.org/10.1007/s11098-009-9389-3

Dorst, C. (2019). Towards a Best Predictive System Account of Laws of Nature. British Journal of Philosophy of Science, 70, 877-900. https://doi.org/10.1093/bjps/axy016

Earman, J., Roberts, J. (2005a). Contact with the Nomic: A Challenge for Deniers of Humean Supervenience about Laws of Nature. Part I: Humean Supervenience. Philosophy and Phenomenological Research, 71(1), 1-22. https://doi.org/10.1111/j.1933-1592.2005.tb00428.x

Earman, J., Roberts, J. (2005b). Contact with the Nomic: A Challenge for Deniers of Humean Supervenience about Laws of Nature. Part II: The Epistemological Argument for Humean Supervenience. Philosophy and Phenomenological Research, 71(2), 253-286. https://doi. org/10.1111/j.1933-1592.2005.tb00428.x

\footnotetext{
${ }^{7}$ Filomeno (2019) desarrolla en detalle un argumento similar contra el humeanismo, sosteniendo que este deja sin explicar las regularidades aparentemente no accidentales observadas en la naturaleza.
} 
Filomeno, A. (2019). Are Non-Accidental Regularities a Cosmic Coincidence? Revisiting a Central Threat to Humean Laws. Synthese, 198(6), 5205-5227. https://doi.org/10.1007/s11229020-02564-9

Hume, D. (1978 [1739]). A Treatise of Human Nature. Oxford: Oxford University Press.

Hume, D. (2000 [1748]). An Enquiry Concerning Human Understanding. Oxford, Oxford University Press.

Jaag, S., Loew, C. (2018). Making Best Systems Best for Us. Synthese, 197, 2525-2550. https:// doi.org/10.1007/s11229-018-1829-1

Jaag, S., Loew, C. (2020). Why Defend Humean Supervenience? The Journal of Philosophy, 117(7), 387-406. https://doi.org/10.5840/jphil2020117723

Ladyman, J., Ross, D., Spurrett, D., Collier, J. (2007). Every Thing Must Go. Metaphysics Naturalized. Oxford: Oxford University Press.

Lange, M. (2000). Natural Laws in Scientific Practice. Oxford: Oxford University Press.

Lange, M. (2009). Laws and Lawmakers: Science, Metaphysics, and the Laws of Nature. Oxford: Oxford University Press.

Lewis, D. (1986). Philosophical Papers, Volume II. New York: Oxford University Press.

Lewis, D. (1994). Humean Supervenience Debugged. Mind, 103(412), 473-490. https://doi. org/10.1093/mind/103.412.473

Loewer, B. (2004). Humean Supervenience. En J. W. Carroll (ed.), Readings on Laws of Nature, pp. 176-206. Pittsburgh: Pittsburgh University Press.

Mackie, J. L. (1980). The Cement of the Universe: A Study of Causation. Oxford: Oxford University Press.

Massimi, M. (2018). A Perspectivalist Better Best System Account of Lawhood. En W. Ott y L. Patton (eds.), Laws of Nature, pp. 139-157. Oxford: Oxford University Press.

Psillos, S. (2002). Causation and Explanation. London: Acumen.

Soto, C., Bueno, O. (2019). A Framework for an Inferential Conception of Physical Laws. Principia: An International Journal of Epistemology, 23(3), 423-444. https://doi.org/10.5007/1 808-1711.2019v23n3p423

Soto, C., Rodríguez, P. (2019). Capacidades y leyes fenomenológicas: el disposicionalismo experimental. Revista de Filosofía, 76, 185-201. http://dx.doi.org/10.4067/S071843602019000200185

Soto, C. (2020a). Wigner, las leyes físicas y la efectividad de las matemáticas. Revista Colombiana de Filosofía de las Ciencias, 20(40), 93-127. https://doi.org/10.18270/rcfc.v20i40.3233

Soto, C. (2020b). Some Morals from the Physico-Mathematical Character of Scientific Laws. Trans/Form/Acao: Revista de Filosofía, 43(4), 65-88. https://doi.org/10.1590/01013173.2020.v43n4.04.p65 
Humeanismo y leyes de la naturaleza: alcance y límites

Cristian Soto

Woodward, J. (2003). Making Things Happen. A Theory of Causal Explanation. Oxford: Oxford University Press.

Revista de Humanidades de Valparaíso, 2021, No 17, 145-167

(c) $(1) \Theta$ CC BY-NC-ND 
